\title{
Further studies on the umbilical cord and its water content
}

\author{
J. M. SCOTT ${ }^{1}$ AND R. WILKINSON ${ }^{2}$ \\ From the ${ }^{1}$ Glasgow Royal Maternity Hospital, Rottenrow, Glasgow G4 OSF, and the \\ ${ }^{2}$ Bio-engineering Department, University of Strathclyde, Taylor Street, Glasgow, UK
}

SUMMARY The results show that the varied appearance of the umbilical cord at birth can be related to its water content and that there are associated changes to be seen histologically in all layers of the cord, but especially in Wharton's jelly. Quantimet analysis confirms these findings and highlights a small group where dual pathology may exist. The relationship between oedematous cords, large drip volume, and respiratory distress in the newborn is considered, and a possible explanation for reduced placental transfusion and low red cell mass in these babies is proffered.

At birth the umbilical cord varies quite considerably in appearance and the changes may have a bearing on the progress of the infant. For example, oedema can be a warning of pending respiratory distress or transient respiratory distress, depending on maturity (Coulter et al., 1975), and a thin, wrinkled cord is frequently associated with a light-for-dates or postmature infant (Scott and Jordan, 1972). Wharton's jelly, which surrounds the vessels of the cord, has the properties of a thyxotropic gell (Bacsich and Riddell, 1945) and is capable of holding large amounts of water. It seemed reasonable that much of the change noted above might be related to altered water content. Therefore freeze-drying experiments were designed to investigate this hypothesis. The findings were compared with histological appearance and Quantimet analysis. Since it had been noted that placentae showing cord oedema tended to drip larger amounts of bloodstained fluid over the 24 hours after delivery this fluid was taken for analysis.

\section{Methods}

Four lines of investigation were instituted:

1 Umbilical cords from 57 cases were examined, measured, and graded as before (Scott and Jordan, 1972; Coulter et al., 1975). Diameters were measured at right-angles in the same plane, in segments where vessels wer empty, and the maximum value of the product obtained was regarded as the reading for that cord. Twenty-four were oedematous with diameter-products (DP) of 1.3 or more, and 16 were

Received for publication 28 February 1978 thin and wrinkled with DP less than 0.6. The remaining 17 were normal with DP values in the intermediate range. Sections 3-4 $\mathrm{mm}$ thick were taken from all the measurement sites. These were blotted to remove excess blood, and the percentage water content was estimated using an Edwards Speedivac centrifugal freeze dryer. Test runs showed that a constant weight could be obtained after 24 hours.

2 Placentae from these cords and a further control series of 430 cases, which ranged from 31 to 42 weeks' gestation, having approximately 50 cases in each group, were examined and scored (Scott and Jordan, 1972). The weight of fluid (contained in each plastic bag), which had dripped from the placenta in the 24 hours following delivery, was measured. This was expressed as drip volume (DV) in millilitres and divided by the initial placental weight gave the drip volume ratio (DVR). Labour ward staff were previously warned that the placentae should not be washed before being put in separate plastic bags.

3 Fluid collected from the bags was well mixed, and aliquots were screened spectrophotometrically and by Kleihauer test for fetal and adult haemoglobin. Good correlation was found, and since the Kleihauer result was definitive, this value was used for further analysis. Total haemoglobin estimations were also made. Cases of abruptio placentae, manual removal of the placenta, others with clots weighing more than $10 \mathrm{~g}$, and those with torn cords or broken up decidual surfaces were excluded from the series as it was felt that such complications might interfere with the composition of the fluid in the bag. In all there were 151 cases suitable for examination.

4 All cords on which water content estimations had 
been made were examined histologically. Adjacent blocks were selected; sections were cut at $7 \mu$ and stained by haematoxylin and eosin and for reticulin (Gordon and Sweet). For Quantimet analysis three areas in Wharton's jelly showing maximal oedema were selected, that is, areas showing the most open coarse reticulated pattern. A Quantimet 720 with epidiascope attachment was used, and areas of voids and intercept counts were measured. From this the ratio of area of void to intercept was calculated (A/I). A series of intercept readings at intercept values ranging from 10 to 150 picture points was taken. Quantimet analysis was made independently without knowledge of DP, DVR, or histological assessment, and 28 cords were examined.

\section{Results}

\section{WATER CONTENT}

The results of freeze-drying experiments on 57 umbilical cords are shown in Figure 1. With few exceptions the 24 oedematous cords (group A) stand out distinctly from the rest. These cords had a mean water content of $93.5 \%$; only one case was below $92 \%$ and here the oedema was focal.

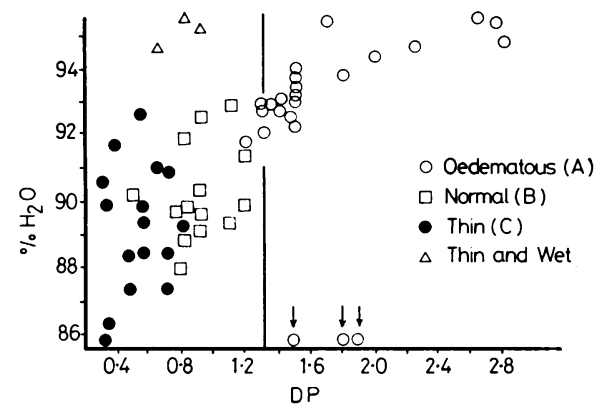

Fig 1 Percentage of water content in 57 blotted sections of umbilical cord compared with diameter product.

Thin wrinkled cords (group C) had a much lower water content, mean $89.2 \%$, but this group could be less readily distinguished from normals (group B) who had a mean water content of $90 \cdot 2 \%$.

Two normal cords and one group $\mathrm{C}$ case had water contents just over $92 \%$, and it was noted that although the DP values were $0.9,1 \cdot 1$, and 0.56 , respectively, Wharton's jelly in each case had a rather wet translucent appearance.

Six cases gave more abnormal findings. These are shown individually in Figure $1(\downarrow)$. Three were grossly oedematous with DP values of $1.5,1 \cdot 8$, and 1.9 . In the cutting blotting process they had obviously lost so much fluid that ridiculously low values were obtained $(84 \cdot 6,36 \cdot 6$, and $68 \%)$.
The three remaining cases $(\triangle)$, which came from group $B$, were complicated, and brief summaries of the clinical findings are shown in Table 1.

Table 1 Clinical findings in three thin wet cords

\begin{tabular}{|c|c|c|c|c|c|}
\hline $\begin{array}{l}\text { Maternal } \\
\text { condition }\end{array}$ & $D P$ & $\begin{array}{l}\% \\
\text { Water } \\
\text { content }\end{array}$ & $D V$ & $D V R$ & $\begin{array}{l}\text { Complication } \\
\text { in baby }\end{array}$ \\
\hline $\begin{array}{l}\text { Anaemia } \\
\qquad \mathrm{Hb} 8.8 \mathrm{~g} / \mathrm{dl}\end{array}$ & 0.80 & 96.06 & 80 & 0.13 & $\begin{array}{l}\text { Cord round neck, } \\
\text { facial congestion } \\
\text { for two days }\end{array}$ \\
\hline $\begin{array}{l}\text { Rhesus (D) } \\
\text { allo-immunisat }\end{array}$ & $\begin{array}{l}0.88 \\
\text { ion }\end{array}$ & $95 \cdot 3$ & 80 & 0.11 & $\begin{array}{l}\text { Exchange } \\
\text { transfusion }\end{array}$ \\
\hline $\begin{array}{l}\text { Twin II } \\
\text { binovular }\end{array}$ & 0.60 & $94 \cdot 57$ & $*$ & * & $\begin{array}{l}\text { Breech. } \\
\text { light-for-dates }\end{array}$ \\
\hline
\end{tabular}

* Not applicable in twin pregnancies.

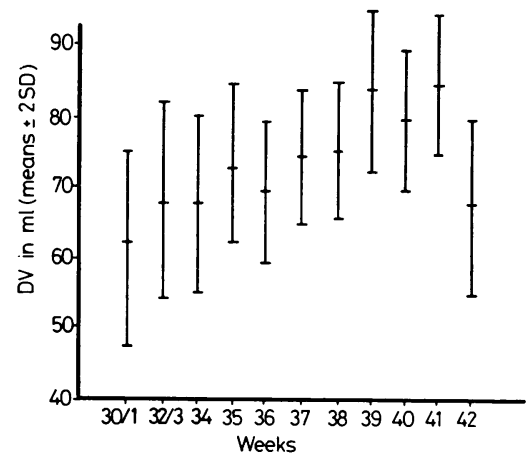

Fig. 2 DV values in 430 control series at various stages of pregnancy.

DRIP VOLUME

Drip volume results from the placentae of these cords and the other $\mathbf{4 3 0}$ cases are shown in Figure 2. Here it can be seen that DV increases from a mean of $62 \mathrm{ml}$ at 31 weeks to $85 \mathrm{ml}$ at 41 weeks but falls thereafter to $66 \mathrm{ml}$ at 42 weeks. Overlap between the different weekly groups is evident but can be related to variations in placental growth. On the other hand, when DVR was examined (Fig. 3) the ratio became constant in the last 10 weeks of pregnancy, mean 0.131 (SD $=0.05, \mathrm{SE}=0.0024)$, providing a standard against which groups $A$ and $C$ and the post-mature at 42 weeks could be compared.

There were 24 cases in group $A$, and in order to increase numbers for statistical analysis another 35 cases with oedematous cords from the larger series of 430 placentae were taken. They also showed increased DV and DVR values, behaving in a similar fashion to the original 24 cases. The mean DVR of this combined group of 59 cases was $0 \cdot 162(\mathrm{SD}=$ 0.046).

Similarly, the original 16 cases in group $\mathrm{C}$ were augmented by another 32 from the larger series, and 


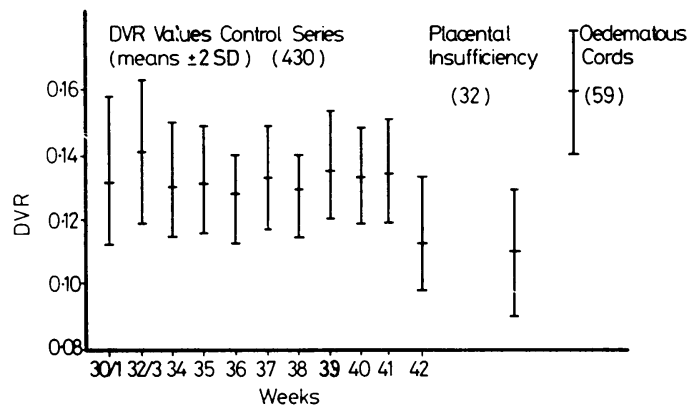

Fig. 3 DVR values in 430 control series compared with groups $A$ (oedematous cords) and $C$ (thin and wrinkled cords of placental insufficiency).

the mean DVR was 0.113 (SD =0.046). Both these groups were then compared with the control series (430 cases) using Student's $t$ test. In group A the difference was highly significant $(t=4.905, \mathrm{P}<$ 0.001 ), but in group $C$ the difference just reached significance $(t=2.546, \mathrm{P}<0.05)$. Nevertheless this group differed significantly from the controls in both placental weight $(P<0.001)$ and DV $(P<0.001)$. The 50 post-mature cases ( 42 weeks, Fig. 2 ) behaved in a similar fashion. They had a low DVR, mean 0.113 , and this just reached significance $(P<0.05)$.

\section{FLUID ANALYSIS}

Of the 151 dripped samples available for analysis, 27 belonged to group A, 85 to group B, and 39 to group C. There was no significant difference in the percentage of fetal cells in each of the three groups, means 35,34 , and $25 \%$, respectively; nor was there any significant difference in haemoglobin levels (mean $5 \cdot 92,5 \cdot 42$, and $6 \cdot 11 \mathrm{~g} / \mathrm{dl}$, respectively). In all three groups the only significant trend noted was the relationship between haemoglobin level and percentage of fetal cells. In group B (85 normals), numbers were sufficient for intragroup analysis, and the results are shown in Table 2 . Here it can be seen that the haemoglobin rose as the percentage of fetal cells increased, and this became highly significant $(P<0.001)$ at levels of $50 \%$ or more.

HISTOLOGY AND QUANTIMET ANALYSIS The various types of umbilical cord have already

Table 2 Group B (85 normals)

\begin{tabular}{lllll}
\hline$\% H b F$ & No. & $\begin{array}{l}\text { Mean } \mathrm{Hb} \\
(\mathrm{g} / \mathrm{dl})\end{array}$ & $S D$ & $P$ \\
\hline$<25$ & 37 & 4.249 & $2 \cdot 183$ & \\
$<50$ & 23 & 5.648 & 1.822 & $<0.05$ \\
$<75$ & 14 & 6.879 & 1.895 & $<0.001$ \\
$<100$ & 11 & 7.709 & 2.356 & $<0.001$ \\
\hline
\end{tabular}

been defined macroscopically (Scott and Jordan, 1972; Coulter et al., 1975), and it was decided to compare and contrast them histologically. All 57 cords were examined as described. Microscopically, the normal cords (group B) showed uniformly small, concentrically arranged spaces in Wharton's jelly, with few broken walls and a normal, evenly distributed cellular content. Group $\mathrm{C}$ cords had few, if any, spaces and more surface clefts (five or more). Very occasionally a cord in this group contains a few larger spaces, irregularly aligned, but generally Wharton's jelly was more condensed and fibrous in appearance, and the reticulin pattern was less open.

Group A showed the most striking changes. Here the spaces were large and irregular, with broken walls and no evidence of alignment. They tended to collect in the angles between vessels and extended out to the periphery in a fan-shaped arrangement. The cellular content in these areas was reduced, the cells becoming small and spindly with pyknotic nuclei. In some very oedematous blebs, swollen cells with vesicular nuclei and eosinophilic cytoplasm could be seen lying free in the spaces. On rare occasions a cord, classified as 'oedematous', had smaller spaces with thickened, rather fuzzy walls. Breaks were fewer in this type of picture. Frequently the oedematous appearance extended to the connective tissue surrounding the vessels and sometimes large spaces were found in muscle coats.

Quantimet analysis was made on 28 of these cords-13 group A, nine group B, and six group C. Intercept readings were taken over a range of 10-150 picture points, and the ratio of void to intercept was calculated at each step. Best discrimination between the three groups was found at an intercept of 120 $\left(I_{120}\right)$, and the results of $A / I$ plotted against $I_{120}$ are shown in Figure 4. Here one can see that the oedematous cords are concentrated in the top righthand corner, that is, they have high values of both $A / I$ and $I_{120}$. Between the other groups there is considerable overlap although once again group $\mathrm{C}$ tended to collect in the lower left-hand corner.

Cases marked 'thin and wet' have been identified in the Figure and these will be discussed later.

\section{Discussion}

The results show that umbilical cords classified as oedematous, with DP values of 1.3 or more, have an increased water content, amounting to at least $92 \%$ wet weight, average $93.5 \%$. At the same time these placentae have an increased dripped volume at 24 hours. In a previous paper (Scott and Jordan, 1972 ) it was suggested that dripped volume was proportional to the size of the intervillous space, provided the decidual surface of the placenta remained 


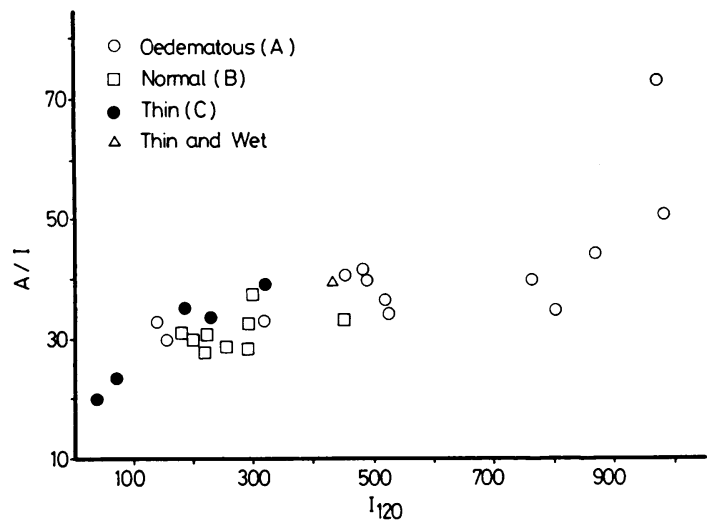

Fig. 4 Ratio of total area to total intercept against number of intercepts greater than 120 picture points $\left(I_{120}\right)$.

intact. The present results add to this and show that, during the last 10 weeks of gestation, DV increased with placental weight but DVR remained constant.

Placentae with oedematous cords (group A) are an exception to this general rule in that they have significantly elevated DV and DVR values. Several factors may account for this. The intervillous space could be bigger initially, or it may be that these placentae fail to contract down to the same extent at the time of delivery. Thus more maternal blood would remain within the placenta and drip out over the next 24 hours.

Alternatively, it could be suggested that the placentae themselves are oedematous and, like hydropic placentae in rhesus allo-immunisation, they drain fluid as well as blood. One would therefore expect to find some alteration in their fluid. Analysis showed, however, that there was no significant difference in the percentage of fetal red cells in the groups, nor was there any significant difference in haemoglobin levels. The only significant trend noted was the link between haemoglobin level and percentage fetal red cells, and this was to be expected because of the higher haemoglobin level in fetal blood.

In group $A$, since there is no alteration in the percentage of fetal cells or in haemoglobin level in the dripped fluid, one must conclude that both fetal and maternal circulations contribute to the increased volume and, as the haemoglobin level showed no significant deviation from normal, excess fluid dripping from the placenta cannot explain the volume increase. Thus one has to revert to the other hypothesis, namely, that these placentae do not retract so well at delivery. If this is so, we have an explanation why these babies have a reduced 'placental transfusion' at delivery, and this may explain the low red cell mass found in these babies with respiratory distress syndrome (Brown et al., 1975).

Failure to retract adequately might be due to extension of the oedematous process to the placenta itself. Certainly connective tissue in stem branches appears to be more oedematous histologically, and this would increase rigidity, allowing more blood to be retained in the intervillous space and villous capillary bed. It is hoped eventually to extend Quantimet analysis to stem branches themselves and if possible provide independent confirmation of this hypothesis. With Quantimet analysis one has to present the computer with a high contrast 'clean' picture. In some cords it was not always possible to obtain the necessary staining density, and errors were greater on area measurement than on $I_{120}$. This can be seen in Fig. 4 where the plot for $I_{120}$ gives a bette: split between the groups than $\mathrm{A} / \mathrm{I}$.

Staining difficulties may account for part of the overlap occurring in the three groups, but one should point out that in each cord measurements were taken on the three most open areas. Thus oedema would be strongly represented in the eventual results, and for this reason thin cords with high values of $I_{120}$ would be identified more readily.

There were three such 'thin wet' cords in the analysis, and these correspond to the group picked out by freeze-drying experiments. In Table 1 it can be seen that additional factors existed in these cases which could lead to water retention. In the first instance there was compression of the cord, in the second anaemia, and in the third a twin pregnancy could have affected water turnover. Thin cords are normally associated with placental insufficiency. They are often wrinkled and, as we have seen, they have a low water content and reduced placental DV and DVR. Light-for-dates babies present a rather similar picture with their dry, wrinkled skin and haemoconcentration (Oski and Naiman, 1972). It seemed inappropriate that an occasional case should show collections of fluid in Wharton's jelly. If one rules out the possibility of local factors, such as cord compression, then general complications such as anaemia, hypoproteinaemia, or even imminent cardiac failure in a light-for-dates baby may have to be considered, and it is interesting to note that this is the type of case where quantitative analysis can be of value in identifying the anomaly. This can be done manually or by automated techniques. To the experienced operator the latter process presents a rapid and easy form of analysis, which in this case was done without prior knowledge of the macroscopic diagnosis or the histological report. Quantimet analysis identified most of the oedematous cords. In a few instances, however, neither histological 
appearance nor Quantimet analysis indicated 'oedema'. Such cords seem to have increased amounts of Wharton's jelly capable of holding excess water. Follow-up of a larger number of babies in this group should prove interesting, especially if they show a differing incidence of respiratory distress.

We acknowledge help with the statistical analysis received from Miss Monica Jordan, of the Department of Bio-engineering, Strathclyde University. Our thanks are also due to Mr Kenneth Tait for technical assistance and to the labour ward staff whose cooperation is greatly appreciated. The Department of Chemistry at Strathclyde University very kindly allowed us to use their Quantimet 720 Image Analyser.

\section{References}

Bacsich, P., and Riddell, W. J. B. (1945). Structure and nutrition of the cornea, cartilage and Wharton's jelly. Nature, 155, 271.

Brown, E. G., Krouskop, R. W., McDonnell, F. E., and Sweet, A. Y. (1975). Blood volume and blood pressure in infants with respiratory distress. Journal of Paediatrics, 87, 1133-1138.

Coulter, J. B. S., Scott, J. M., and Jordan, M. M. (1975). Oedema of the umbilical cord and respiratory distress in the newborn. British Journal of Obstetrics and Gynaecology, 82, 453-459.

Oski, F. A., and Naiman, J. L. (1972). Hematologic Problems in the Newborn, 2nd edition, pp. 78-82. Saunders, Philadelphia.

Scott, J. M., and Jordan, J. M. (1972). Placental in- 응 sufficiency and the small-for-dates baby. American $\dot{\omega}$ Journal of Obstetrics and Gynaecology, 113, 823-832.

Requests for reprints to: Dr J. M. Scott, The Glasgow Royal Maternity Hospital, Rottenrow, Glasgow G4 0NA, UK. 